\title{
Clinical Application of Fecal Drugs in China
}

\author{
Xiaohong Shi ${ }^{1, ~ *, ~ J i a n g l i n ~ Z h a n g ~}{ }^{2}$ \\ ${ }^{1}$ Department of Traditional Chinese Medicine, Affiliated Hangzhou First People's Hospital, Zhejiang University School of Medicine, \\ Hangzhou, China \\ ${ }^{2}$ Department of Clinical Medicine, Zhejiang University School of Medicine, Hangzhou, China
}

Email address:

sxhhzsy@163.com (Xiaohong Shi), jlzhang451613605@sina.cn (Jianglin Zhang)

${ }^{*}$ Corresponding author

\section{To cite this article:}

Xiaohong Shi, Jianglin Zhang. Clinical Application of Fecal Drugs in China. International Journal of Pharmacy and Chemistry. Vol. 4, No. 5, 2018, pp. 35-42. doi: 10.11648/j.ijpc.20180405.11

Received: August 3, 2018; Accepted: September 11, 2018; Published: December 27, 2018

\begin{abstract}
Objective: There is a limited knowledge regarding faecal microbial therapy in modern Western medicine. However, stool has been widely used clinically in the world and it is a relatively mature therapy in ancient China. It has the potential value on the study of gastrointestinal microflora and faecal micro-ecological transplantation. And the clinical experience on stool therapy in ancient China might be useful for the current faecal bacterial transplantation therapy worldwide. Design: The therapy discusses clinical evidences on the use of faeces in ancient China, including donor selection, faeces processing, preservation and its clinical application, including dosage, contraindications and side-effects., but the patients ware not involved. There are faecal transplant breakthroughs on the cognitive. Results: This review describes the different methods of prepareing and processing faecal drugs and the extensive practical treatment applications. It also clarify the ancient techniques for the potential uses of modern faecal medicine. Furthermore, this review will be of interest because of it discusses an emerging area of modern medicine and its bases in traditional Chinese medicine (TCM). Conclusion: The analysis on Natural condition selection, nutritional conditioning and medical evaluation of faecal donor will improve the faecal microflora extraction and promote its mechanism research. It gives us a new sight to understand on distribution of functional bacteria and influencing factors of gastrointestinal microflora. Combined the microflora with equipment, air, soil, additives and foods in the process of faecal processing, it prompt us to re-understand the intestinal nutrition, the faecal microflora and human ecosystem. Meanwhile, the clinical validity of faecal microflora and pathogenesis based on accumulation of clinical practice evidences on the use of faeces in ancient China might be useful for current faecal bacterial transplantation study worldwide.
\end{abstract}

Keywords: Faecal Medicine, Faecal Microflora, Clinical Application, Traditional Chinese Medicine

\section{Introduction}

Fecal medicine was first recorded 3000 years ago in the excavated documents "Collection of 52 Prescriptions" in 1975. That was described as the oldest traditional prescription book in China and buried in No. 3 Han Tombs at Mawangdui. $[1,2]$

A prescription in the book that mixed with hydrargyrum, faecal juice and cinnabar in proportion of $2: 4: 1$, which were took into a closed pot, and roasted with soft fire for three days. Then, it would be made into external form for eliminating the spots and whitening skin, while avoided any cold influence and tire during treatment. [3]

There was the description with faecal juice processing method in the book "Paraphrase of Collection of 52 Prescriptions". In detail, the boy's feces, well water or underground spring water and red soil were put together in winter, which were buried underground for 20-30 years after many processes. At last the juice was divided into three layers and the highest level of juice was needed. It had the effects on removing toxic materials from the body and heat from blood. Some researchers considered the main effective elements of faecal drugs were actinomycetes in red soil, which were the main source of antibiotics which might be produced by fermentation. The side effects of faecal medicine were rarely recorded in Chinese traditional medicine work. [4] 


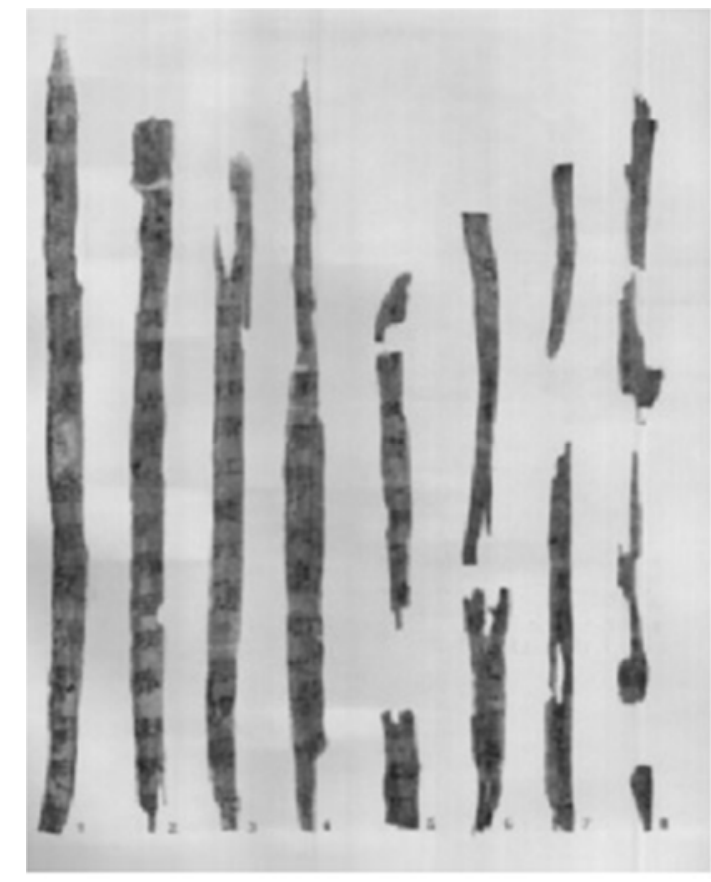

Figure 1. Bamboo slip book.

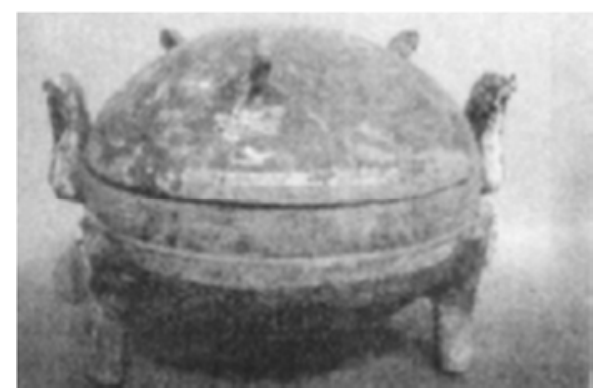

Figure 2. Zhan Dynasty clay pottery in Collection of 52 prescriptions.

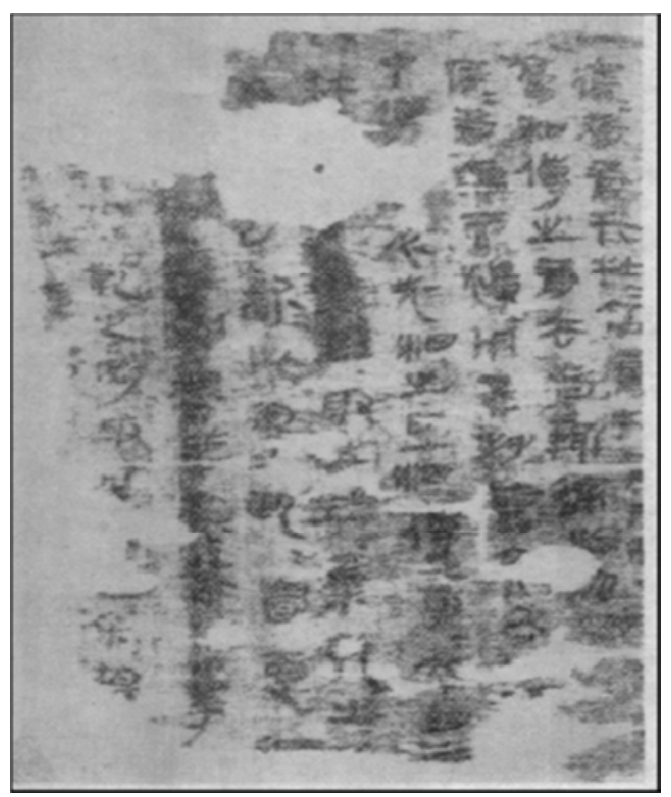

Figure 3. The silk book wan wu.

There were records in "Inter Classic", the activities of ascending, descending, exiting and entering were the basic styles of Qi movement that were responsible for the phenomena of life and death. The Qi movement was dynamic balance between the human microecosystem and external natural environment. Recovering the balance of Qi was the main objective of therapy.

TCM theory stated that the formation of stool and urine was related with the secretion and recognition functions of the small intestine. The strict criteria for ancient doctors to choose uring donors and faecal donors were described in the TCM book "Wan bing hui chun". Five types of donor candidates should be excluded, including impotence; strong voice and rough skin; body or underarm odour; sallow complexion, thin and small corporeity, spado, congenital physical defects and hypophrenia, malnutrition, weak body with easy to get ill; scabies, lepriasis, swelling and ulcers on the body surface, superficial or deep carbuncle. There are also some specific situations for for female donor, namely pubic bone in vulva;, very small of vulva; thickness of hair.

Urine perhaps tonify primordial qi of patients, as a weak body must be deficient in essence, qi, and spirit in traditional Chinese medicine theroy. The age was a key factor for qi, as the primordial qi would be let out in old individuals. The selection factors were describedas follows: the Age should be below 12 or 13-16 years old without an asexual life history, and the appearance should be plump cheek, suitable proportion on face, beautiful eyebrows, bright eyes, florid lips, white teeth, black hair, clear voice and glossy skin. Donors should be provided with a light and clean diet, such as a little salt soup, the food without shallot, ginger, garlic and fragrant-flowered garlic, and also wild goose, dog and tortoise meat.. These donors should be nursed with millet, rice, sorghum, red dates, ormosia, pork, crucian. They should not be allowed to be fatigue to avoid energy dissipation. The living environment, spiritual factors, dietary habits, longevity and gender were also factors should be considered.

Patients were banned to use drugs. Individuals with non-obvious fever symptoms, indigestion, diarrhoea and energy deficiency, as well as those who was cold corpus in nature, those with anaemia and emaciated individuals were excluded, as their primordial qi would be damaged. $[5,6]$ There was a definite record on a therapeutic method, which shows that a litre of faecal juice or soil slurry was taken for poisoning of poisonous fungi and medicine in "Jin gui yao lue" [7] of Zhongjing Zhang about 1800 years ago. (One liter was equal to 200 or 300 milliliters in the Eastern Han Dynasty. [8])

Bangxian Chen, a famous medical historian in China, commented that "Handbook of Prescriptions for Emergency" was compiled based on Que Bian's "Handbook of Prescriptions". (Que Bian, who was a famous doctor, lived 2700 years ago in China. [9])

A clininal case of Hong Ge was recorded in "Medicinal herbs of Dictionary in China". He treated symptom of syncope, vomiting and diarrhoea with horse dung juice extracted by twisting method, which had been identified by Que Bian's experience. Six different feces for clinical application were mentioned in the "Handbook of Prescriptions for Emergency", 
which was written by Ge Hong about 1,700 years ago. Twisting method was also used in the Jin Dynasty and described in "The mixing of urine and human feces". The longer the storage, the better curative effect. [10] Two methods are described in Figures 4 and 5.

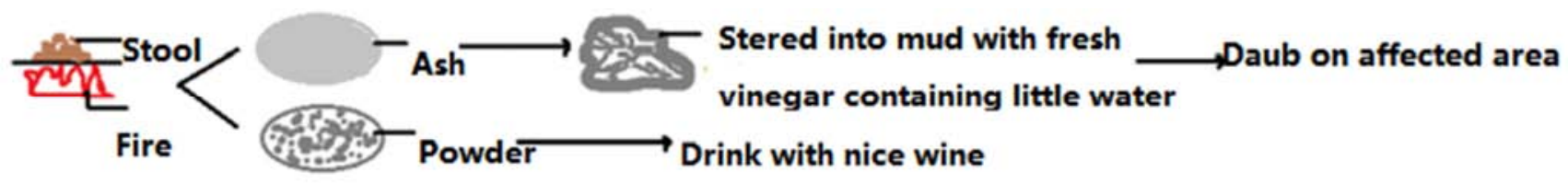

Figure 4. Burning of stools on the fire at different temperatures, which was described in the Jin Dynasty book "Human faeces". Processing scheme was based on "Handbook of Prescriptions for Emergency".

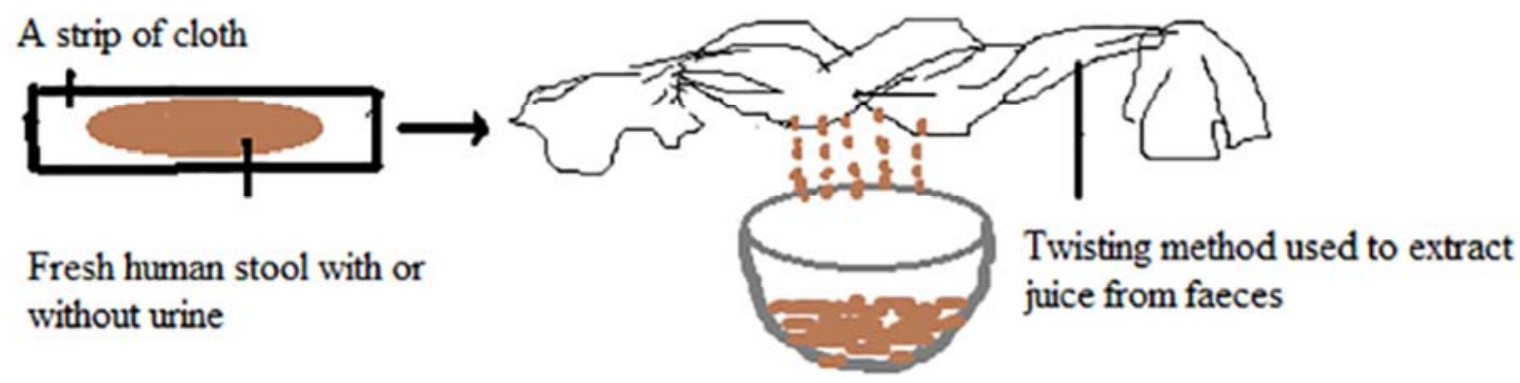

Figure 5. Twisting method written by Ge Hong used in the Jin Dynasty was described in "The mixing of urine and human faeces".

In Liang Dynasty, Hongjing Tao’s “Annotation of Materia Medica" gave a collective way for "Huanglong decoction", In the surrounding area of the city and the temple, the wood plug was utilized to seal clay pottery, followed by steeping it into manure pit. After many years, the pottery would be full of black and bitter faecal juice. In the "Handbook of Prescriptions for Emergency", Hong Ge recorded that if the patient with high fever or the symptoms of nervous system could take drugs, the illness would be alleviated. In the end postscript, the detoxification effect of fecal juice was the best. [11].

\section{Method}

\subsection{Human Faecal Medicine Processing and Clinical Application}

\subsubsection{Dry Human Stool Processing}

The stool was burnt into ashes with a small fire, and the ash was stirred into mud with fresh vinegar containing little water or made into powder to drink with rice wine as described in "Handbook of Prescriptions for Emergency".

In song dynasty, shenwei Tang's "Zheng Lei Ben Cao" [12], the human stool was burnt on the fire until the surface of stool was black, followed by putting the sample into water and stirring it until the water solution was clear, which was drank in the morning. The other way was fry the faeces into red, and take 4 to 5 grams each time orally with wine for 3 times a day. In the Ming Dynasty, a method was descripted to stir a piece of human faeces with the spring or the well water, and gulped it down. Musk and dry human feceswere mixed well with vinegar, the mixture was took for external application.

In the Ming Dynasty, Jiamu Chen [13] had "Diqing" pharmaceutical process. The method was selecting a cool and clean loessland, which was dig a pit about 10-20 centimeters deep, and placed faecal powder and fresh well water into the pit and stirred. The supernatant, called "Di qing", was used to drunk. Shizhen Li's "Ben Cao Gang Mu" in the Ming Dynasty showed that dry human stool in the wild was grinded into powder, which was dipped with ginger slices and ate at 3 to 5 o'clock in the morning. Or the dry human stool in the wild was placed into radish and calcined for about 15 minutes. the sample was took out ground and swallowed with rice wine. Or the dry human stool was smashed with honey and took it orally with the well water that had just been set. Or the dry faece was burnt into ash, which was mixed with bean curd slurry and drank.

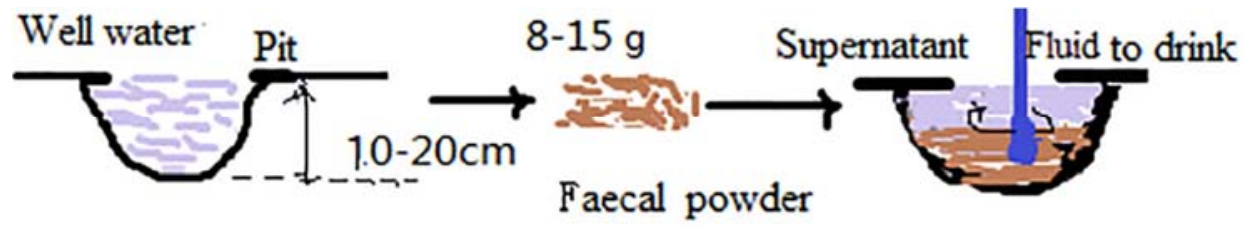

Figure 6. The "Di qing" in the Ming Dynasty was made by placing faecal powder and fresh well water into a yellow soil pit and stirred.

The textbook "medical microbiology and parasitology" recorded that thesoil $10-20 \mathrm{~cm}$ from the ground surface contained a large number of microorganisms, such as actinomycetes, fungi, spirochetes, most of them were beneficial to human.

\subsubsection{Clinical Application}

Human feces's clinical was significant effectively for the 
acute suppurative infection of skin disease, which was wrote in the "Handbook of Prescriptions for Emergency".

In "Ben Cao Jing Ji Zhu", Hongjing Tao said that the human faeces had the better effect for schizophrenia, manic and depression. It was better to take the dry stool powder with warm water that had a good effect for hot syndrome. There was a case in ancient China report that the arrow with poison could lead to human skin damage and die, but the patient would be recovery after drinking fecal juice. While the pigs and dogs did not influenced by the poisonous arrow, due to they often ate feces.

It was suggested that gastrointestinal function played an important role in consciousness, mental state and cognitive function in the long-term clinical practice of Chinese medicine. Faecal drugs had been used to treat psychiatric diseases by restoring the balance of gastrointestinal microbiological.

In the Ming Dynasty, it was recorded that external application of human stool had clinical benefits to abscess under skin and bone tuberculosis, before they were ulcerated and suppurated. The therapy could control local inflammatory manifestations in three days. Acute suppurative disease caused by toxic heat, which was characterized by high fever, was similar to staphylococcus aureus infection, such as meningitis, encephalitis B, epidemic hemorrhagic fever, pneumonia, septicemia with bleeding symptoms. Jaundice, which was the main symptom of disease or was accompanied by liver cirrhosis ascites, could be eliminated with stool medicine after three times treatment. In song dynasty, Shenwei Tang deemed that fresh feces were the most effective drug to treat acute jaundice. Chronic abdominal pain, acute angina pectoralis and the pain caused by all kinds of hernia, gastrointestinal dysfunction, incomplete intestinal obstruction or chronic colon tumefaction resulted in no eating for a long period. It was also used to treat sepsis triggered by acute infectious pediatrics varicella. Human stool were dirty, but the nature was similar to pathogenic factors, so that it was easy to merge into one wholewith pathogenic factors andplay a therapeutic role. "Ben Cao HuiYan" wrotethat human feces were used to cure acute diseases, which had better curative effect than other drugs. A case in the book descripted that the coma patient accompanied by hemi-anesthesia took the ash from stool power mixed with bean curd water, the patient could soon wake up.

The stool had better therapeutic effects on patients with functional low fever, chronic and infectious diseases and some fever diseases caused by unknown causes, which was wrote in the "Herbal Gang Mu" of Shizhen Li in the Ming Dynasty.
The therapeutic range was also suitable for some digestive system diseases, such as dysphagia, functional dyspepsia, belching, gastroesophageal reflux disease, gastritis and dysphagia. TCM theory believed that the pathogenesis of dysphagia was qi stagnation, phlegm obstruction, blood stasis, abnormal esophageal motility, adverse rise of stomach qi and body fluid dried-up not to moisten esophagus. Human faeces's nature was extremely cold. It could eliminate not only fire in an excess or deficiency condition, but also eliminate blood and phlegm stasis. The time of taking medicine was $3 \mathrm{am}$ to $5 \mathrm{am}$. According to the operation time of meridian in TCM, it was the period of circulation with Lung Meridian of Hand-Taiyin. The lung meridian originated from the spleen and stomach of the middle Jiao, connected to the large intestine, circled with cardia of stomach and passed through the diaphragm, ended with the lung. The meridian qi was strong and the ability to resist evil was strong at $3 \mathrm{am}$ to $5 \mathrm{am}$, so this period to take drug had a better effect than the other time.

In song dynasty, shenwei Tang's "Zheng Lei Ben Cao" recorded that human feces were used to treat genital ulcer or bedsore of children, women postpartum uterus and vaginal prolapse.

In the period of the Republic of China, Cunren Chen's "Dictionary of Chinese pharmacology" cited physician's experience in tang dynasty. Human feces had been used to treat skin mucocutaneous and surgical disease with serious damage and infectious, including sore and ulcer caused by external or wind-heat pathological factors. The treatment was very effective. Nutrients were absorbed and converted into qi and blood after diet entered the stomach, and metabolic waste was convered into feces. The initial state of food had been changed, but it retained the nature of extremely bitter and cold. It had the selective action on Stomach Meridian of Foot -Yangming.

\subsection{Processing and Clinical Application of Fecal Supernatant}

It was well known that the method to produce fecal supernatant was described in "Zheng Lei Ben Cao" in Song Dynasty. A section of green bamboo was collected in winter, the green outer cortex of that section was removed and impregnated into fecal juice, which would permeate into the bamboo. The fecal supernatant in the bamboo could be buried into the soil for one month. After that, the supernatant could be picked out to drink at any time.

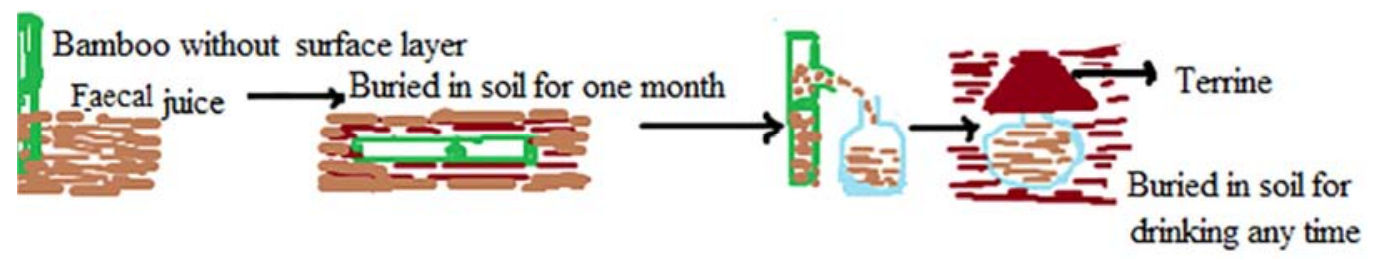

Figure 7. A bamboo branch without rind was placed into faeces to obtain faecal juice.

"Ben Cao Meng Quan" recorded the processing of fecal supernatant. Bamboo baskets were put on the pot, and palm tree bark was laid in baskets, followed by putting layers of thick cotton paper and the fresh soil into the baskets ( 1 ruler in 
Ming Dynasty was equal to 8.1 inches, which was about $31.793 \mathrm{~cm})$. The soil layer was about $19.6 \mathrm{~cm}$. The feces were spread over the soil, so thatthe juice would permeate the basin and poured into a new pottery, which was covered with a rough and unglazed bowl and sealed with mud containing salt water. The whole staff was buried underground for many years. The fecal juice would be as clear as spring water. Some doctors said this processing method was better than the bamboo tube collection method. The longer the time buried of the whole staff, the better therapeutic effect of the fecal juice.

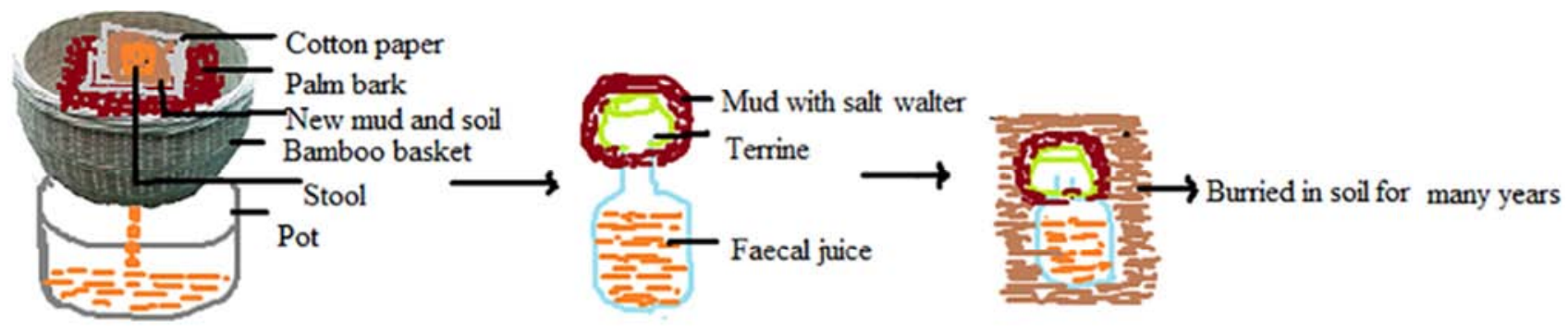

Figure 8. The method in "Ben Cao Meng Quan" to produce fecal supernatant.

The function of fecal supernatant would tend to down, it had no side reaction on the human body due to the medicine originated from the human body. When patients took drugs, transmission route of fecal supernatant still followed its original way and kept the heat and fire pathogenic factors downward. The effect of this therapy was rapid and prominent. "The Dictionary of Chinese Pharmacy" recorded that the properties of medicine were enhanced, the efficacy increased during processing, because it was placed in the soil for a long time. At the initial stage of infantile febrile and infectious disease, the fecal supernatant was effective to dissolve toxic and fire attacking and prevent skin herpes. The contraindication of fecal supernatant was the patient with cold nature, thin and anemia. The theory of fecal supernatant treatment was thought the same as the idea of repairing the broken bamboo with bamboo. It was the best treatment method to eliminate excessive heat in stomach with the stool derived from stomach's food.

\subsection{Ren Zhong Huang}

\subsubsection{Processing method}

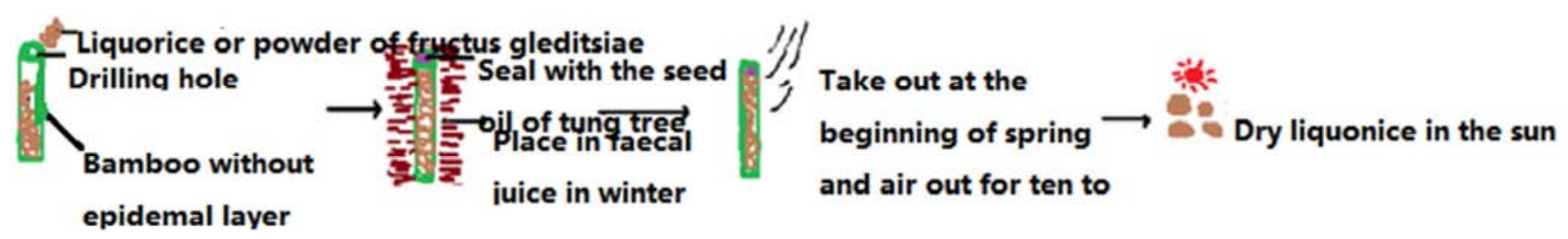

Figure 9. The flow chart of processing "Ren Zhong huang”.

"Ben Cao Meng Quan" recorded that ren zhong huang was fecal processed drug in the Ming dynasty book "Ren Zhong huang", which could be developed with two methods. One was to cut off a section of green bamboo without rind and with one end open, which were filled with pieces of licorice (or honeylocust powder) and sealed with tung oil. After that, the reaction system was put into stool at the beginning of winter and took out in the end of winter, followed byairing it for ten to fifteen days and the liquorice was dried in the sun. The second method was to take a long bamboo without an epidermal layerand one end open, which were also filled with licorice and sealed with a plug. The sealed end of the bamboo was inserted into the feces for one month. The liquorice was took out and dried in the sun to be drug. There was another explanation for "Ren Zhong Huang", which was considered as the yellow condensation accumulated for many years on the brick and stone in the pit of toilet.

\subsubsection{Clinical Application}

"Ben Cao Hui Yan" recorded a case that Ren Zhong huang had obtained good curative effect on the patient with numbness and coma.

The faecal drug fabrication process was often complex. Some reports in modern biochemistry indicated that there was a balance between male and female hormones. In men, the balance was shifted toward the production of androgens, so that most of the oestrogen was excreted in the urine. There was a similar phenomenon for women. Endocrinology studies had confirmed the hormones existed in the stool. Thus, some researchers attempted to isolate sex hormones from stool [14]. Male patients perhaps was treated with urine and faecal medicine derived from women. In fact, male patients absorbed more male hormones, it was beneficial for health. In the same way, female patients obtained female hormones from drugs. In consideration of hormone balance therapy, the selection of faecal medicine was based on sex and age. Therefore, female 
stool may be used to cure male patients and female patients may be treated by using male faeces in the clinical. This therapy warrants deserved to be further studied.

The bamboo, which was the main material in the process of making ren zhong huang, had a white inner membrane. Was it a natural semi-permeable membrane? The principle of treating disease in TCM was "regulating", by reinforcing the healthy qi to eliminate pathogenic factors, eliminate the etiology, restore the normal physiological function of the human body and correct the pathological imbalance. Therefore, whether or not the therapeutic method of ren zhong huang was a special form of microbiological therapy, the valuable experience accumulated by the ancients also deserved to be further studied.

Cunren Chen said that ren zhong huang could be used to treat subcutaneous hemorrhagic diseases. In case of an emergency, soil in a fecal pit could be used. It was worth to point out that modern physicians had also used it to treat various animal diseases. The clinic effect was remarkable. The following examples were the practitioner of TCM verified the therapeutic effect with ren zhong huang to treat diseases.

Xuezhu Zhang [15] treated 28 patients with oral leukoplakia. Ren zhong huang was the constituent part of prescription with 10 grams. The drugs were decocted and took orally combined with gargling for 3-5 minutes and 7 days as a course. The maximum was 4 courses.

Shiming Zhou cured 94 hepatitis B patients with herbal decoction or powder which contained $50 \mathrm{~g}$ ren zhong huang, with by orally for one time a day. The rate of turning negative was 62.65 percent, 59 cases had normal liver function [16]. Erysipelas on the face and parotitis's pathogenic factors were an infectious and epidemic substance in the air, which entered into the body through the nose and mouth. The pathogenesis was based on latent infection of the damp-heat in the spleen and stomach. Danxi Zhu had a prescription named ren zhong huang pills. The main function of the medicine was for epidemic disease. The therapeutic effect of the pills would increase with the increasing storage time. Until the late Qing
Dynasty, Ren Zhong Huang was the most effective medicine for plague, but the production process received bad reviews. Doctors in shanghai believed that it should not be easily denied, which ever had been praised highly by ancient famous doctor, it needed to be improved with modern scientific pharmaceutical technology [17].

Han liang Dong [18] observed therapeutic effect with ren zhong huang to cure hot oral ulcer. Jifang Shao and Yude Jin [19] thoughts that the mechanism of leukemia was toxic pathogens fell into marrow. This "marrow" not only refered to the bone marrow of hematopoietic function system in modern medicine, but also indicated that the depth of the disease was the most serious stage. The treatment method focused on the detoxification. Ren zhong huang could clear blood poison and clean bone marrow. It could relieve symptoms, consolidate curative effect and prolong survival time.

\subsection{Meconium and Children Feces}

It had collected the feces of newborns within 100 days and placed them in a closed container, then removed them for external use after 50 to 60 days recorded in "complementary handbook of prescriptions for emergencies". Also baby dung and lard or water had been used to stir together, feed cow, horse, and donkey. Another method was to collect the feces of male newborn babies, burned them by fire, collected their sublimation, and stored in ceramic container wrote in "complementary collection of compendium of herbology" [20].

\subsection{Meconium and Children's Feces}

\subsubsection{Meconium}

"Medical books of Li shi zhen" [21] showed that it took one month to cure many severe and obstinate skin ulcers, remove polyps, eliminate plot and birthmark with meconium. Drawing out toxin, promoting blood circulation and eliminating spot was also its function.
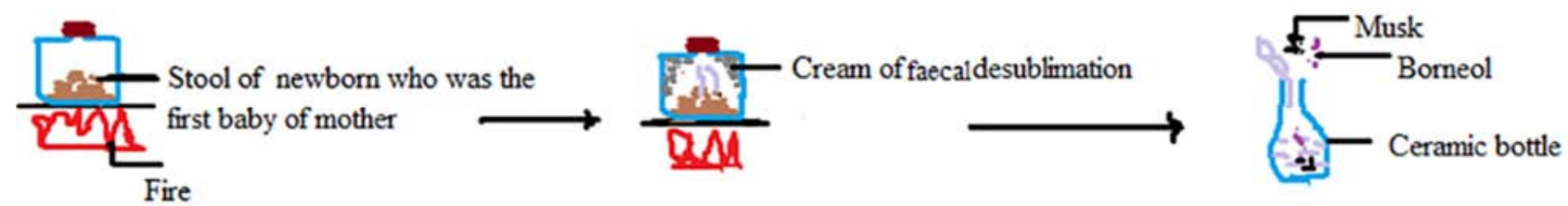

Figure 10. The process for collecting stool from new-born who was the first baby of the mother. The faeces were burnt and the produced cream was placed into a ceramic bottle along with musk and bomeol, as indicated in the book of "Meconium" in the Qing Dynasty.

\subsubsection{Children Feces}

Children stool referred to the feces of infants and children. "Handbook of prescription for emergency" recorded that children's stool was applied on the fistula of surgical abscess. In addition, children's feces and lard were mixed together to treat cattle's abdominal distension. When horse and donkey were pregnant and the urinary tract was blocked, the symptom could also disappear soon with mixture of children's feces and water.

"Zheng Lei Ben Cao" selected children's feces to treat alopecia areata caused by interior deficiency blood associated with exposure to external pathogenic factors. Children's feces, which was different from adult feces, were supposed to nourish blood and hair follicles. In addition, "Complementary collection of compendium of herbology" wrote, a doctor had a prescription of eye drops for sweating. In detail, the newborn male stool, who was the mother's first child, was calcined and took its sublimation to mix with borneol and musk, which was packed with the ceramic container. The usage of the drug was to drop the medicine into left eye (right eye) if the patient was 
a male (female), and milk into the eye and rest for a while.

\subsection{Toilet Paper}

The toilet paper with stool was also drug in "Ben Cao Gang Mu Shi Yi". The 49 pieces of paper with stool was collected and burnt into ashes, which was added two bowls of water. At least a bowl of drug solution was taken orally to cure ka ti cold-induced external contraction. This disease was not identified in "Ben Cao Gang Mu Shi Yi”.

\subsection{White Stool Frost}

It was stool to permeate and congeal at the bottom of the jar recorded in "complementary collection of compendium of herbology". The sample on the jar was scraped and burnt to red with coal, and soaked with vinegar. The process was repeated for nine times. After that, the drugs could be took with wine. It was used to treat the serious condition of bone trauma caused by accidental injury.

\subsection{Rafters on the Toilet}

Rafters on the toilet fumigated by the stools for many years,

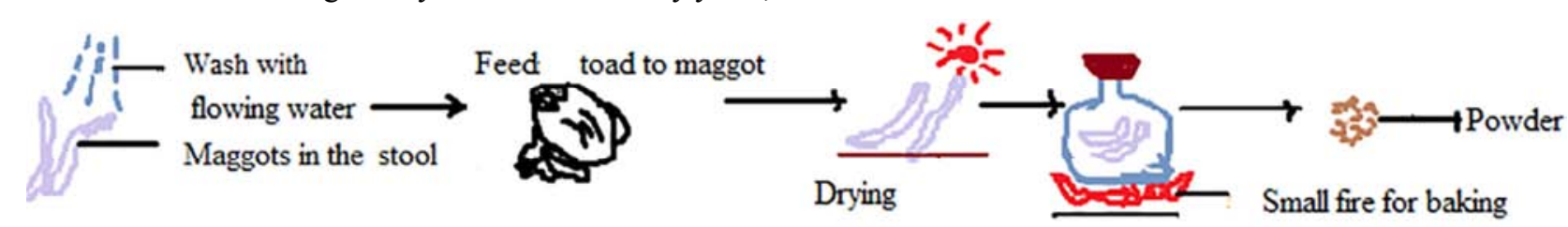

Figure 11. The process on produce feces maggots drug.
The big fecal ascariasis were cleaned cutted off to collect juice.

"Ben cao meng quan"wrote faecal ascariasis came from feces and belonged to cold medicine. According to the record, it could treat liver cirrhosis ascites and jaundice except for feverish disease. The usage was to drop the juice into eyes for treatment of chronic conjunctivitis.

\section{Conclusion}

The formation of feces was accomplished by the joint action of the viscera. Therefore, it contained physiological and pathological information of the whole body. When feces were reabsorbed by gastrointestinal tract, the physiological functions in the body could be strengthened, and also the self-regulating function and immune response mechanism would be further improved. Thus, the healthy qi of eliminating pathogenic factors would be reinforced. The regulation of symbiotic bacteria on the host immune system improved the host's defense. However, the composition and combination of TCM ingredients were quite complex. Pharmacokinetic studies had shown that these component content was very little or undetectable in the blood. At the same time, it was surprising that the bioavailability of many TCMs was very poor. The components of TCMs were rarely detected in blood, tissues and organs, while many western medicines had typical pharmacokinetics characteristics. It was possible that the their function was no less than the fecal supernatant. The broken wood on stool pit would be calcined to ash and ground powder, and stirred them with caramel for treatment of acute tubular lymphangitis by external application.

\subsection{Fecal Maggots and Fecal Ascaris}

"Ben cao meng quan" recorded feces maggots were used as medicine. Feces maggots needed to be washed with flowing water, and fed by toads. The feces maggots was evaporated in the sun, and baked with small fire and pulverization.

"Ben cao meng quan" recorded that fecal maggots had a remarkable effect on treating children with deficiency of qi and blood, gastrointestinal injury and chronic malnutrition characterised by fever, thin, abdominal distension, shortness of breath. "The dictionary of the university of TCM" recorded fecal maggots was nontoxicity, cold in the property, meridian entry of the spleen and stomach. This drug was mainly treated the nervous system symptoms caused by febrile disease and infection of dysentery including bacillary and amoebic dysentery. target of Chinese medicine was intestinal microflora. It perhaps promoted intestinal microecology to turn the pathological state into physiological state through a combination of the TCM ingredients. In this way, the TCMs show a good therapeutic effect on chronic disease and adjust the balance of microecology in the host.

In the long history, Chinese pharmacists and doctors had been studied on faecal drugs and accumulated lots of experience, which was an extremely precious wealth of TCM. It was one of the diagnoses and treatment characteristics to treat diseases with fecal drugs. The extensive and abundant clinical information had provided a large amount of diagnosis and treatment enlightenment for modern medicine, especially the current microbiological transplantation of feces. Using modern biotechnology to develop the therapy of fecal drugs on complex diseases could expand the new concept of feces drug, therapeutic mechanism and novel fecal drug. The development of modern microecology also brought opportunities and prospects for Chinese feces medicine.

\section{Author Contributions}

The paper is the result of joint research, the contribution of every author is comparable to the others. 


\section{Acknowledgements}

I would like to thank my teacher Yunsheng Yang, Chinese PLA General Hospital, and Chinese PLA Medical Academy for his opinions.

\section{Conflicts of Interest}

All the authors do not have any possible conflicts of interest. This work was supported by Administration Office of Zhejiang provincial Traditional Chinese Medicine, No. $2018 Z$ Q040.

No additional data available.

\section{References}

[1] Li ST. Analysis of publication date with Collection of 52 Prescriptions [J]. Correspondence communication of traditional Chinese medicine, 1990, 6:36-37.

[2] Dong SP. Analysis of publication site with Collection of 52 Prescriptions [J]. Journal of traditional Chinese medicine, 1989, 5:12-15.

[3] Ancient Documents Research Office of the Ministry of Culture, Museum of Fuyang Anhui, Research Group for the Han Dynasty bamboo books. The Han Dynasty bamboo books. Cultural Relic. 1975, (9):35-48.

[4] Zhou, D. S., He, Q. H. Paraphrase of Collection of 52 Prescriptions. Shanxi: Shanxi Science and Technology Press, 2013:175-176.

[5] Ming Dynasty, Ni, Z. M., Zheng, J. S., Zhen, X. Y., Yang, M. X. Ben Cao Hui Yan. Beijing: Ancient Works Press, 2005:707.

[6] Qing Dynasty, Wang, A., Chen, T. H. Ben Cao Bei Yao. Beijing, Science and Technology Publishing Press 2012:205.

[7] East Han Dynasty, Zhang, ZJ., Fan, Y. S. Jin Gui Yao Lue. Beijing, Science and Technology Publishing Press 2007:337.

[8] Lu JX, Qiu GM, Qiu L, et al. History of science and technology in China. Beijing, Science and Technology Publishing Press 2001:231.

[9] Chen, MB., Gen JT, Lu ZJ. Record of famous Chinese doctors of in China. Beijing, Science popularization Publishing Press 1987:4-48.
[10] The West Jin Dynasty, Ge, H., Compiling, the Liang Dynasty Hongjing Tao Supplying Shang, ZJ Checking. The Supplement Compiling with Handbook of Prescriptions for Emergency. Anhui, Science and technology publishing press of Anhui. 50, 332 (1983).

[11] Liang Dynasty, Tao HJ, Shang ZJ, Shang YS. Ben Cao Jing Ji Zhu. Beijing, People's Medical Publishing Press, 1994:394-452.

[12] Song Dynasty, Tang, S. W., Guo, S. Q., Jin, X. M. Zheng Lei Ben Cao. Beijing: Science and Technology Publishing Press, 2011:483-484.

[13] Ming Dynasty, Chen, J. M., Zheng, L., Zhao, X. F. Ben Cao Meng Quan. Beijing: China Press of Traditional Chinese Medicine, 2013:273-274.

[14] Li C, Wei FW, Li M et al. History and application of fecal steroid hormone in wildlife research. [J]. Animal in Sichuan, 2003, 22 (4):272-275.

[15] Zhang XZ. Experience in the treatment of oral leukoplakia with traditional Chinese medicine. Medical theory and practice, 2008, 21 (9):1063-1064.

[16] Zhou SM. Herbal decoction of Caoguo and Ren Zhong Huang was used in treatment of 94 cases of Hepatitis B. Traditional Chinese Medicine in Shanxi Province. 1991, 12 (9): 391-398.

[17] Li GG, Yuan FF, Wang H et al. Attach importance to ancient clinical method, which was ancient physicians treating of fever with swollen head. Shanghai journal of traditional Chinese medicine. 2008, 42 (11):59-61.

[18] Dong HL. Clinical application of ren zhong huang, ren zhong bai and animal feces. Chinese Medicine in community. 2005, 21 (13):32-33.

[19] Shao JF, Jin YD. Jin chu zhi's experience in treating leukemia. Chinese Medicine in Jiangsu. 2002, 23 (6): 6-7.

[20] Qing Dynasty, Zhao XM. Ben Cao Gang Mu Shi Yi. Beijing, People's Medical Publishing Press, 1963:368-393.

[21] Ming Dynasty, Li SZ, Hu GC, Liu CH. Medical books of Li shi zhen. Beijing: China Press of Traditional Chinese Medicine, 1963:368-393. 